\title{
Design and Research of Intelligent Mobile Platform
}

\author{
Wang Jiangwei ${ }^{1, \mathrm{a}}$, Liu Hao ${ }^{2, \mathrm{~b}}$,Li Yue ${ }^{3}$, cand Wu Peng ${ }^{4, \mathrm{~d}}$ \\ ${ }^{1}$ North China Electric Power Univercity in Baoding City, Hebei Province, \\ China \\ ${ }^{2}$ North China Electric Power Univercity in Baoding City, Hebei Province, \\ China \\ ${ }^{3}$ North China Electric Power Univercity in Baoding City, Hebei Province, \\ China \\ 4North China Electric Power Univercity in Baoding City, Hebei Province, \\ China \\ a1763737003@qq.com, ${ }^{b} 303145175 @ q q . c o m,{ }^{c} 396131096 @ q q . c o m,{ }^{d} 80$ \\ 6481302@qq.com
}

\begin{abstract}
Intelligent mobile platform realizes intelligent following as well as moving and lifting for height adjustment, and at the same time integrates lots of multimedia equipment including computer, camera, projector, microphone and fingerprint attendance device, which make functions such as assistant teaching for multimedia classroom, exam supervision, review after class and entertainment realized. The platform has various kinds of control methods including the key control, host PC control and mobile phone client control.
\end{abstract}

Keywords:Intelligent following; Height adjustment; Various kinds of control methodsm

\section{Introduction}

With the development of science and technology as well as social progress, the intelligent equipment has been penetrated into every aspect of people's lives. However, the teaching equipment in classrooms for the popularization of scientific knowledge cannot catch up with the time. According to the survey, it is 
found that there are many inconveniences in the process of using the cabinet teacher's desk in the classroom now. On one hand, its height is fixed, so it cannot meet different requirements of teachers; on the other hand, when using the computer or other multimedia equipment, the teacher is restricted to the range surrounding the platform, and cannot go to students' places. But now the development direction of classroom is toward the research and communicating types, which needs the teacher and students to have more interaction and communication, and a mobile platform can provide great convenience for it. The intelligent mobile platform designed and manufactured by us, has the function of following the teacher through the indoor tracking and localization technology, is equipped with a computer, projector, camera, microphone, fingerprint identification instrument and other multimedia devices, and combines the functions such as intelligent following, height adjustment, mobile projection, fingerprint attendance, speech recorder, and video supervision, etc., solving the hardware limitation for the promotion of research class.

\section{Present Foreign and Domestic Research}

At present, there are two main directions for the improvement of the platform both at home and abroad. Firstly, the platform is installed with walking wheels, which needs to be pushed by people. It is not equipped with multimedia equipment and only for the convenience of carriage, so the function is single. Secondly, the integration of the existing multimedia equipment is applied to make the teacher more convenient in the use of multimedia equipment, through the development of assistant software. However, the two methods of improvement cannot solve the main problem that the activity range of the teacher is limited. The intelligent mobile platform developed by us, not only realizes following the teacher to move, and expanding the activity range of the teacher, but also integrates the necessary multimedia equipment, and has the humanistic assistant software, and more abundant function. 


\section{The System Structure and Working Principle of Intelligent Mobile Platform}

\subsection{System Structure}

The main structure of platform includes five parts such as the mobile device, lifting device, multimedia devices, control devices, and following and localization device;
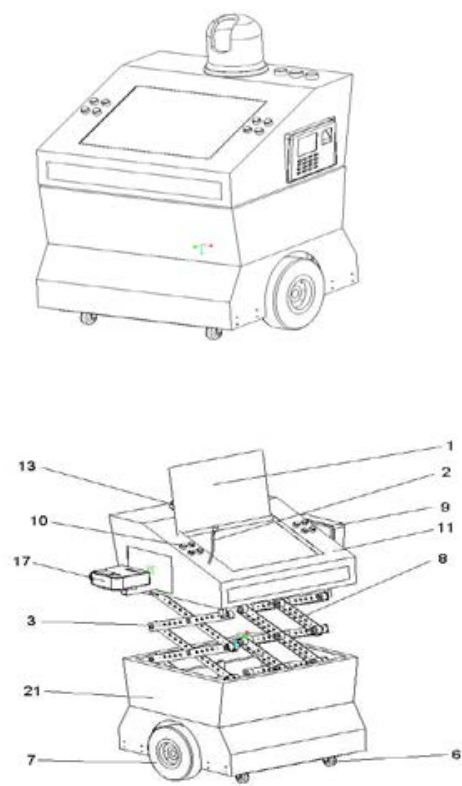

\subsubsection{Mobile Device}

The mobile device is an extendable two-wheeled walking mechanism. The mobile platform has two mobile modes, including the human pushing and motor drive. The whole walking mechanism is installed on the chassis rack, and consists of the driving motor, push-bar motor, and wheel carrier. The two motor cabinets are connected into a whole wheel carrier through a connecting rod. The wheel carrier is connected with the extendable push-bar motor by the connecting rod and the extendable push-bar motor is hinged to the chassis rack through a shaft. Thus the extendable push-bar motor and wheel carrier can rotate around the shaft. The lifting part is realized through the process that the driving wheel is driven by an expansion link (push-bar motor) to rotate around a certain shaft, and 
the push-bar motor is similar to a hydraulic cylinder.

\subsubsection{Lifting Device}

Intelligent mobile platform can realize the elevation and falling through the lifting parallelogram mechanism, uniquely uses angle aluminum as the material, and opens pore in diagonal aluminum skeleton in the premise of ensuring the mechanical strength, which makes the whole device high in strength and light in weight. The elevation and falling of push-bar motor can drive the left shear fork column and right shear fork column to rise and fall, and a lifting push-bar motor provides power for the lifting push-bar mechanism. There is limit switch in the lifting push-bar motor, so as to ensure that the motor will not be stuck to damage the drive circuit.

\subsubsection{Multimedia Devices}

(1) The voice processing part: The camera and microphone are connected to the computer, and controlled by PC software, to realize the video recording and sound amplifying function.

(2)Fingerprint attendance and mobile projection section:Through the connection between HDMI type standard video interface and laptop, mini projector module has realized mobile projection, which turns the walls of the classroom to projection screens, making it convenient for students majoring in Art Design and construction to communicate with teachers, and enriching the instructional mode in class. Fingerprint attendance module can compare the fingerprint input by dean's office with the current attendance fingerprint data, and then preserve the result to local EXCEL document. After signed in, it can directly carry out information check on the Internet, and dump and analyze the EXCEL document reserving the attendance result out of USB.

\subsubsection{Control Device}

The master control device of the platform is STM32 singlechip. STM32 singlechip will take charge in receiving information sent by button, phone Bluetooth, and host computer (PC), controlling the platform to act according to the direction. 


\subsubsection{Tracking and Positioning Device}

There is a WiFi signal transmitter module installed in every direction of the platform, and they four are installed to the same horizontal plane. WiFi module is installed inside a fan-shaped metal enclosure, which makes the WiFi signal transmit in the fan area that appears small. Therefore, signal right ahead of the WiFi module and lateral signal are of great difference. We design phone software to detect the signal strength of the four WiFi modules. The constraint function from the fan makes the differences of the four signal strength detected on the same area become large, thus it can accurately calculate the relevant position of the phone and the platform. Achieved the relevant position between the two, it can realize platform and camera's track to teachers. When the platform can follow the teacher automatically, its mobility can really make sense. And it can make teachers enter into students, and control the multimedia and demonstrate on the platform all the time.

\subsection{Control Modes of Intelligent Mobile Platform}

There are three types concerning control modes of intelligent mobile platform: Physical Button Controler, Computer Software Controler, and Phone Software Controler.

\subsubsection{Physical Button Controler}

When buttons are installed on the control panel, it can realize the controllation on platform by sending signals to master control singlechip, which makes the operation simple and convenient.

\subsubsection{Design of Host Computer Software}

Host computer software sends massages to singlechip through serial port, which mainly contains three functions. First, control the platform to complete the movements as walk and rise and fall; second, control the video information transcribed by camera and microphone, and upload it to the campus local area network; third, compare the fingerprint collected by fingerprint identification instrument with the one in the data base, generating class attendance form and uploading to the campus local area network, which can update with the new 
fingerprint when connected to the campus network.

\subsubsection{Phone Bluetooth Real Time Controler}

We developed phone client for platform, so when applying it, users will only have to install out client on their phones, and then they can control the platform through phones. When the phone sends messages through follower module, Bluetooth module will send messages to singlechip through serial port. It is not only economic but convenient for teachers to use.

There are two control modes in applying the phone software.

(1)Touch Button: Favorable operation interface will rescue users thoroughly, by which they can complete corresponding actions through only one gentle press;

(2)Voice Controler: utilizing the voice identification function of the phone can be more sensitive than normal voice identification module; and the voice module which is separated from the platform makes the voice controler become more flexible.

\subsection{Work Modes of Intelligent Mobile Platform}

Intelligent mobile platform contains two types of work patterns: Class Teaching Mode, Exam Supervision Mode.

\subsubsection{Class Teaching}

In class, the platform enters into teaching mode. Firstly, sign in work attendance.Then,when the class formally begins, the platform rises and then the teacher can freely control the lifting and movement of the platform. When multimedia equipment starts up, the teacher can control the projector by means of computer. The platform automatically follows the teacher and the projector can arbitrarily choose the position of projection, which are convenient for teachers and students to exchange. The camera and the voice device on the platform will record the whole teaching process, which facilitates students to review.

Now many teachers use multimedia in teaching lectures, so they have to 
stay beside the desk in most of the lecture time. Even if they are able to use wireless mouse which only realizes click function, they still cannot carry out real-time operation and demonstration, thus limiting the activity scope of teachers, so they cannot achieve good interaction with students and their teaching quality is affected either. The intelligent follow-up walking, contraction and lifting functions of the mobile platform enable the teacher to get rid of restrictions of the fixed desk, facilitate teachers to make lectures and help to improve classroom teaching quality.

\subsubsection{Exam Supervision}

The platform is adjusted to supervision mode in exams. Students' fingerprints data is stored in fingerprint identification machine, so when they make fingerprints identification, the screen can show whether their input fingerprints match with the fingerprints in the database. After fingerprint identification has been completed, the platform can automatically patrol according to the terrain imported in advance, move to distribute test papers in the classroom, carry on the examination room patrol, record the whole test process with camera and enjoy double degree of freedom platform with no dead angle. It also can implement the supervision function in examination by testing the cellphone signal, so once someone is using a cell phone, it will alarm in time.

\section{Analysis of Future Application}

Most technologies that mobile platform uses have already been mature at present, so the equipment cost is not high. The platform realizes the multi-function operation and greatly improves the cost effectiveness through the integrated use of much equipment. Its application can save a lot of human and material resources, and the saving cost is greater than its cost, so it is possible to realize large-scale popularization and application.

Intelligent mobile platform can solve many disadvantages in the classroom, which enables the teacher to get rid of the platform restrictions, meets the needs of teachers and students to improve the efficiency in class and can be promoted and used in various schools. At the same 
time, the application of the platform is not just limited in the classroom teaching, it can also be used at the venue in which many lectures are frequently hosted or interview sites. It enjoys wide application scope, easy operation, simple maintenance and high comprehensive cost effectiveness.

\section{References}

[1] Ma Chao. Principle and Application of AVR Single Chip Embedded System. Beijing: Beihang University Press, August, 2011.

[2] Siegwart,R. Nourbakhsh,I.R.Autonomous Mobile Robots. Xi'an: Xi`an Jiaotong University Press, 2006;

[3] Zhang Yongqing. Mechanical Design. Beijing: Publishing House of Electronics Industry, 2014;

[4] Lu Jingchao. Automatic Control Theory. Xi'an: Northwestern Polytechnical University Press, (9)2004;

[5] Ou Qingli, He Kezhong. Development of Intelligent Mobile Robot and Research of Key Techniques, Robot. 2000-11, 22(6). 